\title{
Asthma Clinics in Primary Healthcare Centres in Oman \\ Do they make a difference?
}

Abdulaziz Al-Mahrezi, ${ }^{1}$ Sawsan Baddar, ${ }^{2}$ Sheikha Al-Siyabi, ${ }^{3}$ Safaa Al-Kindi, ${ }^{3}$ "Ibrahim Al-Zakwani, ${ }^{4}$ Omar Al-Rawas

$$
\text { عيادات علاج مرض الربو في مراكز الرعاية الصحية الأولية في عمان }
$$

$$
\text { عبد العزيز المحرزي، سوسن بدّار، شيخه السيابية، صفاء الكندية، إبراهيم الزكواني، عمر الرواس }
$$

ABSTRACT: Objectives: This study aimed to determine the effect of newly established asthma clinics (ACs) on asthma management at primary healthcare centres (PHCs) in Oman. Methods: This retrospective crosssectional study was conducted between June 2011 and May 2012 in seven PHCs in the Seeb wilayat of Muscat, Oman. All $\geq 6$-year-old asthmatic patients visiting these PHCs during the study period were included. Electronic medical records were reviewed to determine which clinical assessment and management components had been documented. Results: A total of 452 asthmatic patients were included in the study. The mean age was $35 \pm 21$ years old (range: 6-95 years) and the majority (57\%) were female. In total, 288 (64\%) cases were managed at ACs and 164 (36\%) were managed at general clinics (GCs). Significant differences were noted in the documentation of cases managed at ACs compared to those at GCs, including history-taking information regarding signs and symptoms (91\% versus $19 \% ; P<0.001)$, trigger factors ( $79 \%$ versus $16 \% ; P<0.001)$ and a history of atopy $(81 \%$ versus $17 \% ; P<0.001)$, smoking $(61 \%$ versus $7 \% ; P<0.001)$, asthma exacerbations $(73 \%$ versus $10 \% ; P<0.001)$ or previous admissions (63\% versus $10 \% ; P<0.001)$. Furthermore, prescription rates of inhaled corticosteroids (72\% versus $61 \%$; $P=0.021)$ and short-acting $\beta$-agonists $(93 \%$ versus $82 \% ; P=0.001)$ were significantly higher at ACs compared to GCs. Conclusion: Overall, the findings indicated that ACs have had a positive impact on asthma management at the studied PHCs.

Keywords: Asthma; Disease Management; Patient Compliance; Medical History Taking; Medical Records; Documentation; Oman.

الملخص: الههف: تحديد أثر عيادات الربو المنشأة حديثاً لعلاج الربو في مراكز الرعاية الصحية الأولية في عمان. الطريقة: تم عمل تدليل

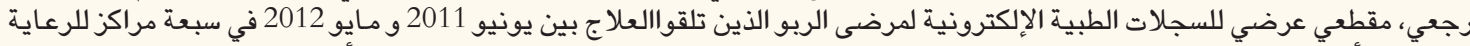

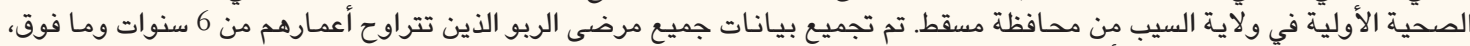

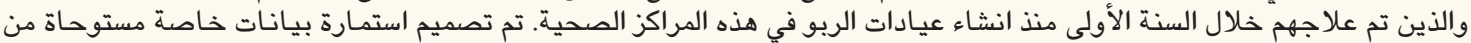

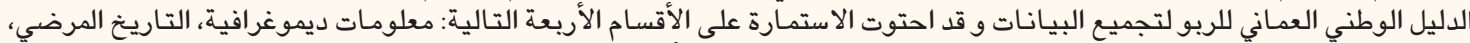

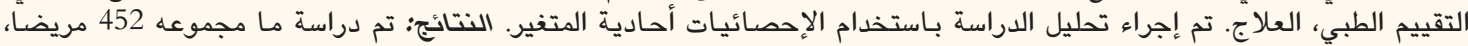

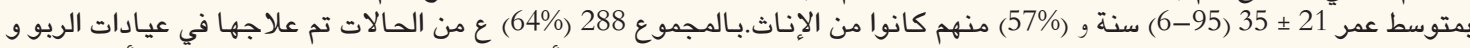

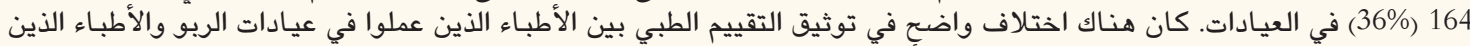

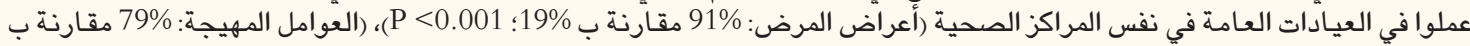

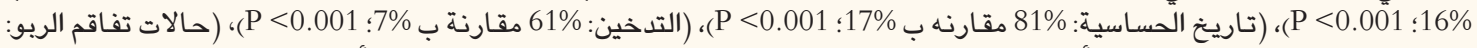

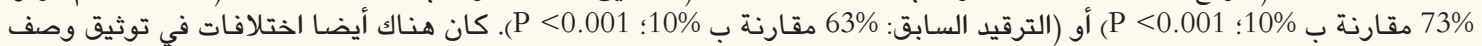

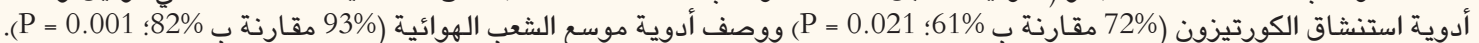
الخلاصة: تشير نتائج الدراسة إلى الأثر الإيجابي لتأسيس عيادات الربو في مراكز الرعاية الصحية الأولية الهية.

الكلمات المفتاحية: الربو؛ علاج المرض؛ مواظبة المريض؛ تسجيل التاريخ الطبي؛السجلات الطبية؛ التوثيق؛ عمان.

\section{Advances IN KNOWLedge}

This study found that the management of asthmatic patients at newly established asthma clinics (ACS) in Oman resulted in significantly greater documentation of various clinical assessment and management components compared to cases seen at general clinics.

\section{Application to Patient Care}

The findings of this study highlight the need for the establishment of more specialised ACs in Oman so as to improve management protocols and patient outcomes. 
A STHMA IS A COMMON CHRONIC DISEASE AND a major cause of morbidity worldwide. ${ }^{1-4}$ It is a challenging disease that requires a systematic approach as, if not appropriately controlled, the condition can have negative consequences for patients, their families and the healthcare system. ${ }^{5-8}$ Physicians are expected to possess the essential knowledge and skills to continually assess, monitor and adjust management plans in order to prevent future exacerbations and achieve satisfactory asthma control. ${ }^{9}$ However, several studies have indicated that most children and adults do not have well controlled asthma and do not comply with recommended treatment regimens. ${ }^{10-14}$

Previous research has also highlighted poor adherence to asthma care protocols and the insufficient documentation of various management components among general practitioners (GPs). ${ }^{15-19}$ Training healthcare providers, providing them with necessary resources and encouraging adherence to standardised management protocols can lead to a significant improvement in asthma control and reduction in management costs. ${ }^{20-23}$ Moreover, the introduction of nurse-led asthma clinics (ACs) has been shown to be cost-effective, with similar clinical outcomes to cases managed at physician-led clinics. ${ }^{24}$

In Oman, patients with acute complaints can receive medical care at any time at a network of primary healthcare centres (PHCs). Each PHC has specialised clinics for diabetes, hypertension, antenatal and immunisation care and is staffed by one or two family physicians as well as GPs from various medical backgrounds. However, the majority of asthmatics are managed by different physicians and at different clinics. ${ }^{19}$ In 2009 , the second edition of the National Asthma Guidelines was released and made available to PHC physicians; subsequently, a programme was launched to establish specialised ACs in PHCs in the Muscat governorate in 2010..$^{25,26}$ In addition, all PHCs in the Muscat governorate also have access to essential resources required to manage mild and moderate asthma cases, such as peak flow metres, spacer devices and asthma medications, including salbutamol inhalers with longacting or short-acting $\beta$-agonists (SABAs), inhaled corticosteroids (ICS), oral and intravenous corticosteroids and theophylline. ${ }^{19}$

In line with the National Asthma Guidelines, the main aim of the $\mathrm{AC}$ programme was to improve the management of asthmatic patients in PHCs by training healthcare providers on the practical aspects of asthma management, including both theoretical and practical sessions on the management of asthmatic children and adults. ${ }^{25,26}$ However, subsequent research conducted in 2010 revealed no immediate improvement in asthma management following the launch of the national guidelines. ${ }^{19}$ Therefore, the aim of this study was to compare asthma management between cases seen at the newly established ACs and those at general clinics (GCs) within the same PHCs.

\section{Methods}

This retrospective cross-sectional study was conducted between June 2011 and May 2012 at seven PHCs located in the Seeb wilayat of Muscat governorate, including Al-Koudh, Al-Shadi, Al-Seeb, Al-Mawaleh North, Al-Mawaleh, Al-Maabela North and Al-Maabela South clinics. All of these ACs were newly established at some point during the study period. All patients aged $\geq 6$ years old with mild or moderate asthma-related symptoms visiting the PHCs during this period were included in the study. However, patients who presented with acute asthmatic exacerbations requiring emergency management, those who were diagnosed with other chronic respiratory diseases and patients requiring only repeat prescriptions or receiving routine care at other healthcare institutions were excluded. The sample size was calculated to be 387 patients based on an average prevalence of $50 \%$ (range: 2-77\%) of complete documentation of management components by GPs in GCs and assuming the difference in documentation completion by GPs in ACs to be at least $10 \% .{ }^{19}$ The alpha and power values were maintained at $5 \%$ and $80 \%$, respectively.

Subsequently, at each of the seven PHCs, the electronic medical records of 100 consecutive asthmatic patients were reviewed and included in the study. A data collection form derived from the National Asthma Guidelines was utilised. ${ }^{26}$ The information collected consisted of demographic characteristics and the type of clinic visited; in addition, various history-taking (i.e. signs and symptoms of asthma, trigger factors and a history of atopy, smoking, asthma exacerbations or previous admissions), clinical assessment (i.e. peak expiratory flow rate [PEFR], level of asthma control, inhaler technique and patient compliance) and management (i.e. prescription of ICS, inhaled SABAs and antihistamines and a scheduled date for the next follow-up appointment) components were analysed and marked as either documented or undocumented according to the contents of the records. Documentation was considered complete if both positive and negative information was recorded (e.g. inability to use a peak flow metre, satisfactory inhaler technique, etc.) and considered incomplete if the required component was missing. So as to avoid bias, every PHC was given a unique identification code and medical staff at the centres were not involved in the data collection process. 


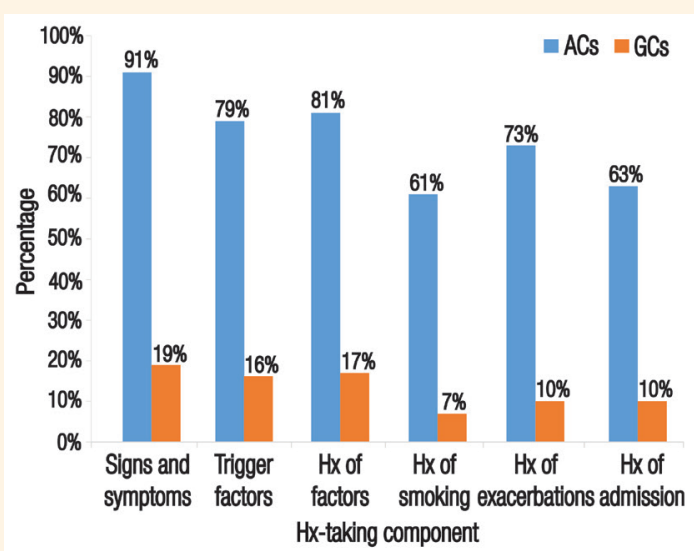

Figure 1: Chart showing the percentage of complete documentation of various history-taking components in the medical records of asthma cases seen at asthma clinics versus general clinics in Seeb wilayat, Oman $(\mathrm{N}=452)$. $A C s=$ asthma clinics; $G C s=$ general clinics; $H x=$ history.

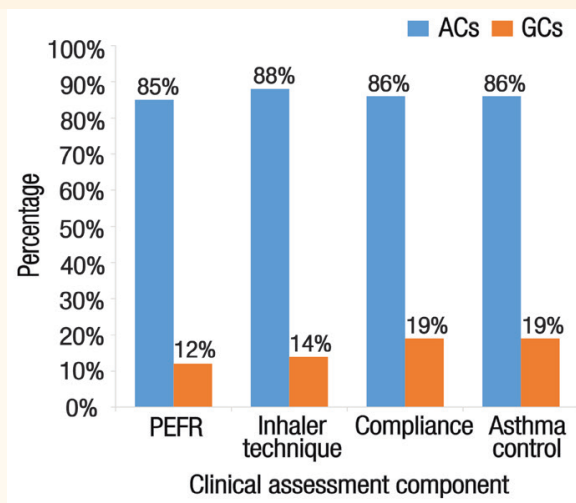

Figure 2: Chart showing the percentage of complete documentation of various clinical assessment components in the medical records of asthma cases seen at asthma clinics versus general clinics in Seeb wilayat, Oman $(\mathrm{N}=452)$.

$A C s=$ asthma clinics; $G C s$ = general clinics; $P E F R=$ peak expiratory flow rate.

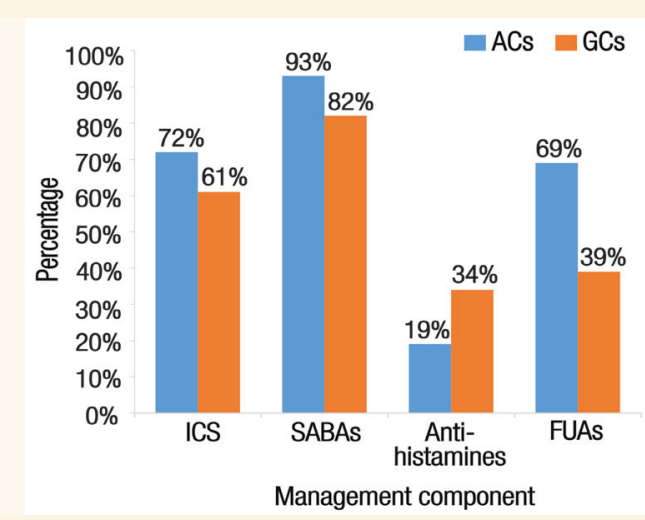

Figure 3: Chart showing the frequency of prescriptions and follow-up appointments for asthma cases seen at asthma clinics versus general clinics in Seeb wilayat, Oman $(\mathrm{N}=452)$.

ACs = asthma clinics; GCs = general clinics; ICS = inhaled corticosteroids; $S A B A s=$ short-acting $\beta$-agonists; FUAs = follow-up appointments.
The data were analysed using Stata ${ }^{\circledR}$ data analysis and statistical software, Version 13.1 (StataCorp LLC, College Station, Texas, USA). Descriptive statistics were used to describe the data, with frequencies and percentages reported for categorical variables and means and standard deviations for continuous variables. Medians and interquartile ranges were used to present non-normally distributed data. Differences between the AC and GC groups were analysed using Pearson's Chi-squared test or Fisher's exact test for categorical variables and the Student's t-test or Mann-Whitney U test for continuous variables, as appropriate. An a priori two-tailed level of significance was set at $P \leq 0.050$.

Ethical approval for this study was obtained from the Research and Ethical Review and Approval Committee of the Directorate of Primary Health Affairs, Ministry of Health, Oman (\#MH/DG PS/33).

\section{Results}

A total of 452 patients were seen by 74 GPs at the PHCs during the study period. The overall mean age of the patients was $35 \pm 21$ years old (range: 6-95 years) and 256 (57\%) were female. In total, 288 (64\%) cases were managed at ACs and 164 (36\%) were managed at GCs. Overall, 87 patients (19\%) were managed by GPs who worked only at the ACs, 84 (19\%) by GPs who worked only at the GCs and 281 (62\%) by GPs who worked at both types of clinics. Significant differences were noted in the documentation of clinical historytaking, assessment and management components for cases managed at ACs and GCs.

Overall, physicians at ACs were significantly more likely to complete history-taking documentation compared to those at GCs, including information regarding signs and symptoms (91\% versus $19 \% ; P<0.001$ ), trigger factors $(79 \%$ versus $16 \%$; $P<0.001)$ and a history of atopy $(81 \%$ versus $17 \% ; P<0.001)$, smoking $(61 \%$ versus $7 \%$; $P<0.001)$, asthma exacerbations $(73 \%$ versus $10 \% ; P<0.001)$ or previous admissions (63\% versus $10 \%$; $P<0.001$ ) [Figure 1]. Moreover, documentation regarding the clinical assessment of the patient's inhaler technique (88\% versus $14 \%$; $P<0.001$ ), asthma control ( $86 \%$ versus $19 \%$; $P<0.001)$, treatment compliance $(86 \%$ versus $19 \%$; $P<0.001$ ) and PEFR (85\% versus $12 \% ; P<0.001$ ) was significantly higher among cases managed at ACs compared to those managed at GCs [Figure 2]. In terms of management, cases managed at ACs had significantly higher prescription rates of ICS (72\% versus $61 \% ; P=0.021)$ and inhaled SABAs (93\% versus $82 \% ; P=0.001$ ) compared to GC cases. However, physicians at GCs were significantly more likely to prescribe antihistamines than physicians at ACs (34\% 
Table 1: Documentation completeness of asthma cases seen by the same doctors working at both types of clinics* at asthma clinics versus general clinics in Seeb wilayat, Oman $(\mathrm{N}=281)$

\begin{tabular}{|c|c|c|c|}
\hline \multirow[t]{2}{*}{ Component } & \multicolumn{2}{|c|}{$\begin{array}{c}\text { Documentation, } \\
\mathbf{n}(\%)\end{array}$} & \multirow[t]{2}{*}{$P$ value } \\
\hline & $\begin{array}{c}\text { Cases } \\
\text { seen at } \\
\text { ACs } \\
(n=201)\end{array}$ & $\begin{array}{c}\text { Cases } \\
\text { seen at } \\
\text { GCs } \\
(\mathbf{n}=80)\end{array}$ & \\
\hline Signs and symptoms & $178(89)$ & $53(66)$ & $<0.001$ \\
\hline Trigger factors & $152(76)$ & $18(23)$ & $<0.001$ \\
\hline History of smoking & $106(53)$ & $8(10)$ & $<0.001$ \\
\hline History of atopy & $156(78)$ & $17(21)$ & $<0.001$ \\
\hline $\begin{array}{l}\text { History of asthma } \\
\text { exacerbations }\end{array}$ & $142(71)$ & $10(13)$ & $<0.001$ \\
\hline $\begin{array}{l}\text { History of previous } \\
\text { admissions }\end{array}$ & $130(65)$ & $10(13)$ & $<0.001$ \\
\hline PEFR & $166(83)$ & $12(15)$ & $<0.001$ \\
\hline Compliance & $173(86)$ & $15(19)$ & $<0.001$ \\
\hline Inhaler technique & $176(88)$ & $15(19)$ & $<0.001$ \\
\hline Asthma control & $173(86)$ & $15(19)$ & $<0.001$ \\
\hline ICS prescription & $147(73)$ & $52(65)$ & 0.046 \\
\hline SABA prescription & $186(93)$ & $66(83)$ & 0.009 \\
\hline $\begin{array}{l}\text { Antihistamine } \\
\text { prescription }\end{array}$ & $36(18)$ & $20(25)$ & 0.120 \\
\hline Follow-up appointment & $114(57)$ & $26(33)$ & 0.046 \\
\hline
\end{tabular}

versus 19\%; $P=0.001$ ). Nevertheless, GPs at ACs were significantly more likely to schedule follow-up appointments (69\% versus 39\%; $P=0.001$ ) [Figure 3].

Among the 281 cases seen by the 25 GPs who worked at both types of clinics (i.e. ACs and GCs), the documentation of all components was significantly greater when the physician worked at the $\mathrm{AC}$ rather than the $\mathrm{GC}(P<0.050$ each), except for antihistamine prescription rates [Table 1]. However, in GCs, no significant differences were noted in documentation completeness when comparing GPs who worked only at GCs and those who worked at both types of clinics [Table 2].

\section{Discussion}

Overall, the current study found that the completeness of documentation of various history-taking, clinical assessment and management components was significantly greater among asthma cases seen at ACs compared to those seen at GCs in selected PHCs in Muscat. This finding might be due to the intensive training that most physicians received prior to the establishment of the ACs; alternatively, it could be due to the structured management approach followed at these clinics. Previous research has indicated that
Table 2: Documentation completeness of asthma cases seen at general clinics (GCs) by doctors working either only at $\mathrm{GCs}^{*}$ or at both types of clinics ${ }^{\dagger}$ in Seeb wilayat, Oman $(\mathrm{N}=164)$

\begin{tabular}{lccc} 
Component & \multicolumn{2}{c}{$\begin{array}{c}\text { Documentation, } \\
\text { n(\%) }\end{array}$} & P value \\
& $\begin{array}{c}\text { Cases seen } \\
\text { by doctors } \\
\text { at both } \\
\text { types of } \\
\text { clinics } \\
(\mathbf{n}=\mathbf{8 4})\end{array}$ & $\begin{array}{c}\text { Cases } \\
\text { seen by } \\
\text { GC-only } \\
\text { doctors } \\
(\mathbf{n}=\mathbf{8 0})\end{array}$ & \\
& $50(60)$ & $53(66)$ & 0.421 \\
& $13(15)$ & $18(23)$ & 0.319 \\
Signs and symptoms & $4(5)$ & $8(10)$ & 0.239 \\
Trigger factors & $11(13)$ & $17(21)$ & 0.213 \\
History of smoking & $6(7)$ & $10(13)$ & 0.298 \\
History of atopy & $7(8)$ & $10(13)$ & 0.268 \\
History of asthma & & $12(15)$ & 0.343 \\
exacerbations & $8(10)$ & $15(19)$ & 1.000 \\
History of previous & $16(19)$ & $15(19)$ & 0.116 \\
admissions & $8(10)$ & $15)$ & 1.000 \\
PEFR & $16(19)$ & $15(19)$ & \\
Compliance & $48(57)$ & $52(65)$ & 0.339 \\
Inhaler technique & $68(81)$ & $66(83)$ & 0.842 \\
Asthma control & $36(43)$ & $20(25)$ & 0.120 \\
ICS prescription & $37(44)$ & $26(33)$ & 0.150 \\
SABA prescription & & &
\end{tabular}

$G C=$ general clinic; $P E F R=$ peak expiratory flow rate; $I C S=$ inhaled corticosteroids; SABA = short-acting $\beta$-agonist .

*A total of 37 doctors worked only at GCs. ${ }^{\dagger} A$ total of 25 doctors worked at both types of clinics.

the management of asthmatic patients is easier and more effective when GPs are guided by a standardised management protocol. ${ }^{27}$ However, a study in Oman reported that junior GPs had greater documentation rates compared to junior pulmonologists, despite both groups following the same management protocol. ${ }^{28} \mathrm{~A}$ previous study comparing asthma management in two different settings within the same healthcare institution reported better documentation of symptoms by residents compared to medical officers (64\% versus 35\%). ${ }^{19}$ To the best of the authors' knowledge, the current study is the first to assess differences in documentation among the same GPs working in two different settings.

Both control and preventative medications are important in order to achieve better control of the disease and improve the quality of life of asthmatic patients. In this regard, ICS are considered the cornerstone of management and should be prescribed to all asthmatic patients upon discharge. ${ }^{12,29}$ In this study, ICS were prescribed to $72 \%$ and $61 \%$ of patients managed at ACs and GCs, respectively. In contrast, Arnold et al. found that only $47 \%$ of hospitalised adult asthmatic patients had received ICS prior to 
admission, potentially causing asthma exacerbations and subsequent admission to the hospital. ${ }^{18}$ Furthermore, in a study of 100 consecutive children admitted with episodes of acute asthma exacerbation in Kuwait, none of the children were prescribed ICS. ${ }^{30}$ In a tertiary care hospital in Oman, a previous study of all asthmatic patients presenting with acute exacerbations reported that only $33 \%$ of patients had been prescribed ICS in the three months prior to the episode; additionally, this medication was subsequently prescribed after admission to only $58 \%$ of cases. ${ }^{19}$ The same study also showed low levels of therapy-related documentation, with $73 \%$ of patients having no record of a post-nebulisation assessment and $23 \%$ having poor documentation of follow-up appointments-with the latter finding lower compared to those of the current study at both GCs (39\%) and ACs (69\%). ${ }^{19}$ In the current study, when analysing the records of patients seen by GPs who worked at both ACs and GCs, the documentation of follow-up appointments was higher when patients were seen at the AC rather than the GC ( $57 \%$ versus $33 \%$, respectively).

A relative lack of consultation time could account for the differences in management components noted between the two types of clinics in the current study. Generally, GPs who work in GCs are expected to attend to more patients with a much wider range of conditions compared to those in ACs. In addition, both nurses and pharmacists were included in the intensive training programme prior to the launch of the ACs in Muscat and thus expected to be able to assess a patient's PEFR, inhaler technique and asthma control without the assistance of a physician. Consequently, the GPs who worked in the ACs may have had more time to assess patients and document the required information in their medical records. ${ }^{21,24}$ This could also explain the current study's finding that the same doctors performed significantly differently when managing patients seen at ACs and GCs, particularly in the documentation of most analysed components. Moreover, without previous knowledge of the patient's medical history, it can be extremely difficult for a physician seeing a patient for the first time to assess control levels and initiate an appropriate management plan. Therefore, adequate documentation of such information is essential for good clinical practice and high-quality patient care.

The current study is subject to certain limitations. Due to the retrospective and cross-sectional nature of the study, these findings may only be relevant to a specific period of time. Furthermore, this study mainly assessed the documentation of various clinical assessment and management components within the electronic medical records of asthma cases; while this is an essential part of clinical practice and generally expected to reflect greater clinical judgment and better patient outcomes, some doctors may have adequately documented their clinical and management notes but lacked other important clinical skills. It is also possible that some physicians may have performed specific assessment or history-taking actions, but not have documented these steps due to lack of time. However, it is important to highlight that the electronic medical record system used at many PHCs in Oman does not allow physicians to execute certain management actions without the documentation of specific information like prescriptions, referrals and follow-up appointments. It is therefore likely that the electronic records represent a true account of what was assessed or took place during the patient's visit.

\section{Conclusion}

The findings of this study demonstrate the positive impact of newly established ACs at several PHCs in Muscat. Specifically, the documentation of various assessment and management components in the electronic medical records of asthmatic patients was significantly better at ACs compared to GCs. Future studies are recommended to determine whether better documentation is correlated with better patient outcomes and whether management at ACs has an effect on the patient's level of asthma control.

\section{CONFLICT OF INTEREST}

The authors declare no conflicts of interest.

\section{FUNDING}

No funding was received for this study.

\section{References}

1. Global Asthma Network. The global asthma report 2014. From: www.globalasthmareport.org/resources/Global_Asthma_ Report_2014.pdf Accessed: Mar 2018 .

2. World Health Organization. Asthma. From: www.who.int/ mediacentre/factsheets/fs307/en/ Accessed: Mar 2018.

3. Al-Riyami BM, Al-Rawas OA, Al-Riyami AA, Jasim LG, Mohammed AJ. A relatively high prevalence and severity of asthma, allergic rhinitis and atopic eczema in schoolchildren in the Sultanate of Oman. Respirology 2003; 8:69-76. doi: 10. 1046/j.1440-1843.2003.00426.x

4. Al-Rawas OA, Al-Riyami BM, Al-Kindy H, Al-Maniri AA, Al-Riyami AA. Regional variation in the prevalence of asthma symptoms among Omani school children: Comparisons from two nationwide cross-sectional surveys six years apart. Sultan Qaboos Univ Med J 2008; 8:157-64.

5. Al-Busaidi N, Soriano JB. Asthma control in Oman: National results within the Asthma Insights and Reality in the Gulf and the Near East (AIRGNE) study. Sultan Qaboos Univ Med J $2011 ; 11: 45-51$. 
6. Nunes C, Pereira AM, Morais-Almeida M. Asthma costs and social impact. Asthma Res Pract 2017; 3:1. doi: 10.1186/s40733016-0029-3.

7. Masoli M, Fabian D, Holt S, Beasley R; Global Initiative for Asthma (GINA) Program. The global burden of asthma: Executive summary of the GINA dissemination committee report. Allergy 2004; 59:469-78. doi: 10.1111/j.1398-9995.2004. 00526.x.

8. Seung SJ, Mittmann N. Urgent care costs of uncontrolled asthma in Canada, 2004. Can Respir J 2005; 12:435-6. doi: 10. $1155 / 2005 / 478764$

9. Partridge MR. Living with a variable disease. Pulm Pharmacol Ther 2002; 15:491-6. doi: 10.1006/pupt.2002.0399.

10. Al Busaidi N, Al Mukhaini S. Level control of asthma patients in chest specialist clinics. Oman Med J 2009; 24:195-8. doi: 10.5001/omj.2009.38.

11. Al-Rawas OA, Jayakrishnan B, Ben Abid F, George J, Baddar SA, Al-Riyami BM. Management and control of asthma in patients attending a specialist centre in Oman. Sultan Qaboos Univ Med J 2009; 9:132-9.

12. Peters SP, Jones CA, Haselkorn T, Mink DR, Valacer DI, Weiss ST. Real-world Evaluation of Asthma Control and Treatment (REACT): Findings from a national web-based survey. J Allergy Clin Immunol 2007; 119:1454-61. doi: 10.1016/j.jaci. 2007.03.022.

13. Fitzgerald D. Non-compliance in adolescents with chronic lung disease: Causative factors and practical approach. Paediatr Respir Rev 2001; 2:260-7. doi: 10.1053/prrv.2001.0149.

14. Baddar S, Jayakrishnan B, Al-Rawas OA. Asthma control: Importance of compliance and inhaler technique assessments. J Asthma 2014; 51:429-34. doi: 10.3109/02770903.2013.871558.

15. Meng YY, Leung KM, Berkbigler D, Halbert RJ, Legorreta AP. Compliance with US asthma management guidelines and specialty care: A regional variation or national concern? J Eval Clin Pract 1999; 5:213-21. doi: 10.1046/j.1365-2753.1999.00177.x.

16. Finkelstein JA, Lozano P, Shulruff R, Inui TS, Soumerai SB, $\mathrm{Ng} \mathrm{M}$, et al. Self-reported physician practices for children with asthma: Are national guidelines followed? Pediatrics 2000; 106:886-96.

17. Baddar S, Jayakrishnan B, Al-Rawas O, George J, Al-Zeedy K. Is clinical judgment of asthma control adequate? A prospective survey in a tertiary hospital pulmonary clinic. Sultan Qaboos Univ Med J 2013; 13:63-8.

18. Arnold LB, Usery JB, Finch CK, Wallace JL, Deaton PR, Self TH. Inadequate documentation of asthma management in hospitalized adult patients. South Med J 2009; 102:510-14. doi: 10.10 97/SMJ.0b013e31819ecb03.
19. Baddar SA, Al-Ismaili AI, Al-Taie MA, Al-Mahrezi AM, Al-Rawas OA. Documentation of the management of asthma exacerbation in adults by primary health care physicians in a teaching hospital in Oman. Sultan Qaboos Univ Med J 2010; 10:335-40.

20. Cabana MD, Slish KK, Evans D, Mellins RB, Brown RW, Lin X, et al. Impact of physician asthma care education on patient outcomes. Health Educ Behav 2014; 41:509-17. doi: 10.1177/ 1090198114547510

21. Guevara JP, Wolf FM, Grum CM, Clark NM. Effects of educational interventions for self management of asthma in children and adolescents: Systematic review and meta-analysis. BMJ 2003; 326:1308-9. doi: 10.1136/bmj.326.7402.1308.

22. Suh DC, Shin SK, Okpara I, Voytovich RM, Zimmerman A. Impact of a targeted asthma intervention program on treatment costs in patients with asthma. Am J Manag Care 2001; 7:897-906

23. Elward K, Blackburn B, Peterson LE, Greenawald M, Hagen MD. Improving quality of care and guideline adherence for asthma through a group self-assessment module. J Am Board Fam Med 2014; 27:391-8. doi: 10.3122/jabfm.2014.03.130241.

24. Kamps AW, Roorda RJ, Kimpen JL, Overgoor-van de Groes AW, van Helsdingen-Peek LC, Brand PL. Impact of nurse-led outpatient management of children with asthma on healthcare resource utilisation and costs. Eur Respir J 2004; 23:304-9. doi: 10.1183/09031936.03.00052203.

25. Baddar S. Asthma clinic programme at primary health care level. Abstract in: Oman Thoracic Conference 2013: The Oman Respiratory Society and Sultan Qaboos University. Sultan Qaboos Univ Med J 2014; 14:e418.

26. Oman Ministry of Health. Guidelines for the management of Asthma: Second edition, 2009. From: www.moh.gov. om/documents / 272928/1314763/Guidelines +for + management+of+Asthma+Second+edition+2009+\%281\%29.pdf/ c5e8ff0c-d937-4c57-bc2b-d225c26be727 Accessed: Mar 2018.

27. Ting S. Multicolored simplified asthma guideline reminder (MSAGR) for better adherence to national/global asthma guidelines. Ann Allergy Asthma Immunol 2002; 88:326-30. doi: 10. 1016/S1081-1206(10)62016-9.

28. Baddar S, Worthing EA, Al-Rawas OA, Osman Y, Al-Riyami BM. Compliance of physicians with documentation of an asthma management protocol. Respir Care 2006; 51:1432-40.

29. Global Initiative for Asthma. 2017 GINA report: Global strategy for asthma management and prevention. From: https:// ginasthma.org/wp-content/uploads/2016/01/wms-GINA2017-main-report-tracked-changes-for-archive.pdf Accessed: Mar 2018

30. Hijazi Z, Abdulmalek AK, Al-Taweel F, Al-Shareda S. Hospital management of children with acute asthma exacerbations in Kuwait: Adherence to international guidelines. Med Princ Pract 2002; 11:126-30. doi: 10.1159/000063244. 